\title{
Review of: "CDC50 orthologues in Plasmodium falciparum have distinct roles in merozoite egress and trophozoite maturation"
}

Prapon Wilairat ${ }^{1}$

1 Mahidol University

Potential competing interests: The author(s) declared that no potential competing interests exist.

Manuscript title: CDC50 orthologues in Plasmodium falciparum have distinct roles in merozoite egress and trophozoite maturation

Authors: Avnish Patel, Stephanie D. Nofal, Michael J. Blackman and David A. Baker

\section{General comment}

Plasmodium falciparum contains guanylyl cyclase alpha (GC $\alpha$ ), a type III integral membrane protein whose production of cGMP is required for merozoites egress from host red blood cells through activation of PKG. GC $\alpha$ is a bifunctional protein with a cyclase domain located at endo-C-terminal region and ecto-N-terminal P4-ATPase domain (with putative flippase activity). In model organisms P4-ATPases require interaction with cell division control protein 50 (CDC50) for their flippase activity. P. falciparum genome contains three CDC50 orthologues ( $\mathrm{A}, \mathrm{B}$ and $\mathrm{C}$ ), with the latter two expressed in the intraerythrocytic stage. Employing gene tagging and conditional gene disruption, together with immunofluorescence analysis (IFA), immunoprecipitation (IP) and mass spectrometry (MS), the authors demonstrate that CDC50B binds GC $\alpha$ to stimulate optimal CGMP synthesis for normal merozoite egress; while CDC50C is a binding protein of another putative P4-ATPase, ATP2, which, interestingly, is involved in cytostome-mediated uptake of host hemoglobin into the parasite acidic vacuole for proteolytic globin degradation and detoxification of released heme through a hemozoin biocrystallization process.

This work is an important preliminary investigation into the (surprisingly) neglected study of a class of malaria parasite membrane phospholipid transporters [flippase (transport from outer (exoplasmic) to inner (cytoplasmic) leaflet, floppase (from cytoplasmic to exoplasmic leaflet) and scramblase (exchange between the two leaflets)], all of which are involved in membrane remodeling required for such cellular processes as endocytosis, exocytosis, vesicle formation (from intracellular ER/golgi and extracellular budding from plasma membrane), and autophagy.

\section{Specific comments}

Comment 1. It is not clear in the context of this manuscript why the CDC50s should be named 
"chaperones", as they clearly act as binding partners of P4-ATPases. A "chaperone protein" is defined as a protein that delivers a protein "under its care" to its final location or assists in appropriate folding, and then dissociates.

Comment 2. The merozoite membrane localization of both $C D C 50 B$ and $C D C 50 C$ as demonstrated by IFA would be more convincing if the magnification/resolution of the immunofluorographs (Fig 1E) were improved. This also applies to Fig 4D.

Comment 3. In Fig 3A, the authors should provide an explanation for the weak bands observed in the "input" lanes. In Fig 3B, the green spots are not clearly visible; suggest using black spots. It is not clear the reason behind the locations of red spot designating CDC50-B (recommend change to "CDC50B") and CDC50C. Data on fragment size and \% coverage of the cognate protein should be included in a Supplementary Table, otherwise readers will have to believe the data depicted in Fig 3B based on blind faith.

Comment 4. As merozoite egress is an important aspect of the work, the authors should have included another egress assay to that of measurement of the proteolytically processed form of SERA5, e.g., measurement of new ring formation or parasitemia at $\sim 36$ hours post-egress.

Comment 5. The fluorescent lipid labelling experiment does not contribute to the main findings of the work. As the fluorescent lipids (NBD-PC, -PE and -PS) would be incorporated into infected (i)RBC membrane, it would be expected that any increase in phospholipid content of iRBC membrane would not have any effect on parasite lipid uptake, irrespective of the presence or absence of CDC50B and CDC50C. It would have been better to use saponin-treated iRBC.

Comment 6. In Fig 5B, "count" on y-axis should be explained in the figure legend.

Comment 7. It is notoriously difficult to quantify hemozoin content in situ. It would have helped if it could be shown that null CDC50C parasites were more sensitive to chloroquine or dihydroartemisinin than controls.

Comment 8. On lines 310-313, the authors refer to the work of Lamy A, et al. (ref. 48) who reported the interaction between $\mathrm{CDC} 50 \mathrm{~B}$ and ATP2. The authors should comment on the absence of this interaction in their study.

Comment 9. In Discussion, it might be useful if a final paragraph be included to indicate the future research directions stemming the findings and how the work impacts the current status of our understanding of malaria parasite membrane phospholipid transporters (flippases).

\section{Minor comments.}

Minor comment 1. On line 183, 190 and 197, "Fig 4E", "Fig 4F" and "Fig 4G" should read "Fig 4D", "Fig $4 \mathrm{E}$ " and "Fig 4F", respectively.

Minor comment 2. In Fig 1C, the headings of the left gel should be shifted so that they are located above their respective lane; in right gel " $\mathrm{C}$ " is missing above $2^{\text {nd }}$ lane from left.

Minor comment 3. In Fig 1E, "Merge + DAPI" should have "+ DAPI" located below "Merge". This also 
applies to Fig 4D.

Minor comment 4. In Fig 4C, in left panel the symbol "*" should be located to the right of lane "Beads boil".

Minor comment 5. In Fig 4F, the format size of the lane headings should be adjusted so that the headings are located above their cognate lane.

Minor comment 6. In Fig 6E, " $\alpha$ Haemoglobin" should read " $\alpha$ Globin".

Minor comment 7. "CDC50-B/C" should read "CDC50B/C". 